\title{
Global Stabilization of the Oscillating Eccentric Rotor
}

\author{
CHIH-JIAN WAN, DENNIS S. BERNSTEIN*, and VINCENT T. COPPOLA ** \\ Department of Aerospace Engineering, The University of Michigan, Ann Arbor, MI 48109-2118, U.S.A.
}

(Received: 15 April 1994; accepted in revised form: 21 February 1995)

\begin{abstract}
The oscillating eccentric rotor has been widely studied to model resonance capture phenomena occurring in dual-spin spacecraft and rotating machinery. This phenomenon arises during spin-up as a resonance condition is encountered. We consider the related problem of rotor despin. Specifically, we determine nonlinear feedback control laws that not only despin the rotor but also bring its translational motion to rest. These globally asymptotically stabilizing control laws are derived using partial feedback linearization and integrator backstepping schemes. For the case in which the oscillating eccentric rotor is excited by a translational sinusoidal forcing function, the control law is shown to attenuate the amplitude of the translational oscillation.
\end{abstract}

Key words: Passage through resonance, cascade system, integrator backstepping.

\section{Introduction}

We are ultimately interested $\mathbf{i}$ i controlling dual-spin spacecraft; however, in this paper, we analyze a model that embodies similar dynamical behavior: the oscillating eccentric rotor. The control problem involving dual-spin spacecraft is concerned with reducing nutation that becomes excited during spin-up. The interaction between spin and nutation has been modelled in [12] by means of the oscillating eccentric rotor where translation represents nutation. The interaction between rotation and translation in the oscillating eccentric rotor is analogous to the interaction between spin and nutation in dual-spin spacecraft.

The oscillating eccentric rotor consists of an unbalanced rotor attached to an elastic support. This system has been used as a simplified model to study the resonance capture phenomenon in dual-spin spacecraft during spin-up under small constant torque $[4,7,12,15]$. The capture phenomenon represents the failure of a rotating mechanical system to be spun up by a torquelimited motor to a desired terminal state due to its resonant interaction with another part of the system $[3,12]$. In $[3,12]$, the dynamics of the oscillating eccentric rotor were analyzed using perturbation theory, while in [7] control laws for rotor spin-up under limited torque were obtained and extended to dual-spin spacecraft.

In this paper, we consider the oscillating eccentric rotor but, rather than spinning up the rotor, we seek feedback control laws that globally asymptotically stabilize the entire system, both rotation and translation, to the rest state using only a torque actuator. Aside from potential application to dual-spin spacecraft, such control laws for the oscillating eccentric rotor are of independent interest. For example, the controlled oscillating eccentric rotor serves as a rotational actuator for suppressing translational vibration [2].

The synthesis technique used to obtain globally asymptotically stabilizing control laws involves partial feedback linearization $[1,10,11]$ and integrator backstepping for cascade systems $[1,6,8,13]$. In particular, we apply partial feedback linearization to transform

\footnotetext{
* Research supported in part by the Air Force Office of Scientific Research under Grant F49620-92-J-0127.

** Research supported in part by NSF Grant MSS-9309165.
} 


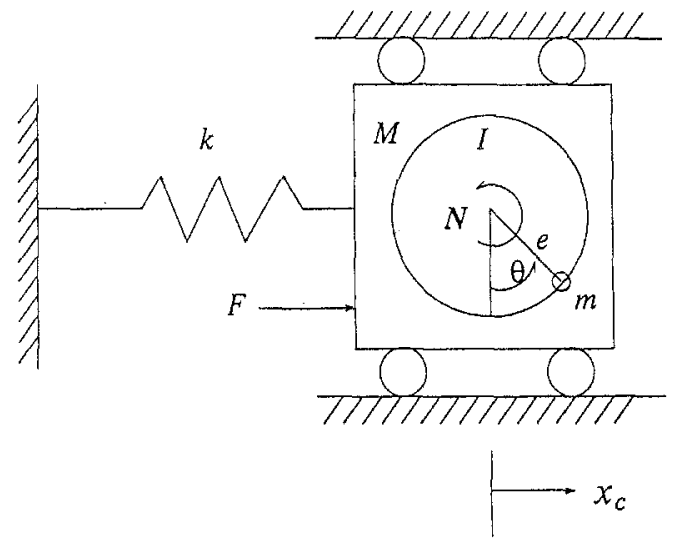

Fig. 1. Oscillating eccentric rotor.

the dynamical equations of the oscillating eccentric rotor into a system in cascade form. Then we derive stabilizing control laws for the system in cascade form using integrator backstepping. Control laws for the original system are then obtained by applying the reverse transformation.

\section{Dynamic Model of the Oscillating Eccentric Rotor}

The oscillating eccentric rotor shown in Figure 1 consists of an unbalanced point mass of mass $m$ attached to a rotary disk of inertia $I$ which is riding on a cart constrained to move horizontally. The moving cart is attached to a wall by a spring with spring constant $k$. Let $M$ denote the total mass of the disk and the cart, and let $e$ denote the distance between the center of the disk and the unbalanced mass. For simplicity, we assume that the motion is confined to the horizontal plane so that there are no gravitational effects. Let $x_{c}$ denote the translational position of the center of the disk from its equilibrium position, and let $\theta$ denote the counter-clockwise rotational angle of the unbalanced mass where $\theta=0$ corresponds to a 90-degree rotation from the spring axis as shown in Figure 1. Let $N$ denote the control torque applied to the disk and let $F$ denote a translational disturbance force applied to the moving cart. Note that $I=0$ is allowed, that is, the disk may be massless.

The equations of motion for the system are given by $[3,7,12,15]$

$$
\begin{aligned}
& (M+m) \ddot{x}_{c}+m e\left(\ddot{\theta} \cos \theta-\dot{\theta}^{2} \sin \theta\right)+k x_{c}=F, \\
& \left(I+m e^{2}\right) \ddot{\theta}+m \ddot{x}_{c} e \cos \theta=N .
\end{aligned}
$$

Introducing the dimensionless variables [7]

$$
\begin{aligned}
x_{d} \triangleq \sqrt{\frac{M+m}{I+m e^{2}}} x_{c}, \quad \varepsilon \triangleq \frac{m e}{\sqrt{\left(I+m e^{2}\right)(M+m)}}, \\
u \triangleq \frac{(M+m)}{k\left(I+m e^{2}\right)} N, \quad \tau \triangleq \sqrt{\frac{k}{M+m}} t, \quad F_{d} \triangleq \frac{1}{k} \sqrt{\frac{M+m}{I+m e^{2}}} F,
\end{aligned}
$$

equations (1) and (2) are equivalent to the dimensionless equations

$$
\ddot{x}_{d}+x_{d}=\varepsilon\left(\dot{\theta}^{2} \sin \theta-\ddot{\theta} \cos \theta\right)+F_{d},
$$




$$
\ddot{\theta}=u-\varepsilon \ddot{x}_{d} \cos \theta
$$

where the dot and double dot now denote first and second derivatives with respect to $\tau$. By defining $x=\left(x_{1}, x_{2}, x_{3}, x_{4}\right)^{T}=\left(x_{d}, \dot{x}_{d}, \theta, \dot{\theta}\right)^{T},(3)$ and (4) can be written in first-order form as

$$
\dot{x}=f(x)+g(x) u+d(x) F_{d},
$$

where

$$
\begin{aligned}
& f(x) \triangleq\left[\begin{array}{c}
x_{2} \\
\left(-x_{1}+\varepsilon x_{4}^{2} \sin x_{3}\right) /\left(1-\varepsilon^{2} \cos ^{2} x_{3}\right) \\
x_{4} \\
\varepsilon \cos x_{3}\left(x_{1}-\varepsilon x_{4}^{2} \sin x_{3}\right) /\left(1-\varepsilon^{2} \cos ^{2} x_{3}\right)
\end{array}\right], \\
& g(x) \triangleq \frac{1}{1-\varepsilon^{2} \cos ^{2} x_{3}}\left[\begin{array}{c}
0 \\
-\varepsilon \cos x_{3} \\
0 \\
1
\end{array}\right], \quad d(x) \triangleq \frac{1}{1-\varepsilon^{2} \cos ^{2} x_{3}}\left[\begin{array}{c}
0 \\
1 \\
0 \\
-\varepsilon \cos x_{3}
\end{array}\right] .
\end{aligned}
$$

Note that the denominator $1-\varepsilon^{2} \cos ^{2} x_{3}$ is never zero, since, by definition, $\varepsilon<1$. It is easy to check that if $F_{d}=0$ and $u=0$, then every equilibrium state $f(x)=0$ of (5) is of the form $x_{1}=x_{2}=x_{4}=0$ with arbitrary $x_{3}$, which corresponds to zero translational position and velocity, zero angular velocity, and arbitrary rotation angle $x_{3}=\theta$ of the unbalanced mass. In this paper we are interested in the equilibrium state corresponding to $x_{3}=0$. In particular, we are interested in deriving globally asymptotically stabilizing control laws for the equilibrium state $x=0$.

\section{Cascade System Control Synthesis}

We now consider a single-input general nonlinear affine control system of the form

$$
\dot{x}=f(x)+g(x) u,
$$

where $x \in \mathbb{R}^{n}, u \in \mathbb{R}$, and we assume $f(0)=0$. In the case $F_{d}=0$, the oscillating eccentric rotor model (5) is a special case of (6) with $n=4$. Our goal is to find a feedback control $u=u(x)$ that globally asymptotically stabilizes (6) to $x=0$. This is accomplished by means of a two-step procedure. First we transform (6) to a the simpler form

$$
\begin{aligned}
& \dot{\zeta}=\phi(\zeta, \xi), \\
& \dot{\xi}=v,
\end{aligned}
$$

using partial feedback linearization $[1,10,11]$, where $\phi(\zeta, \xi)$ is $C^{1}, \zeta \in \mathbb{R}^{n-1}$ and $\xi, v \in \mathbb{R}$ Having transformed (6) into the form (7) and (8), we then seek control laws to stabilize (7) and (8). Control laws for the original system (6) can then be obtained by using the reverse transformation.

As shown in [13], the cascade form of (7) and (8) is convenient for stabilization. Specifically, if (7) can be stabilized by viewing $\xi$ as the control input, then the combined system (7), 
(8) can be stabilized by means of a suitable function $v=v(\zeta, \xi)$. This procedure, which is known as integrator backstepping $[6,8]$, is based on the following result obtained by Sontag and Sussmann [13] and Kokotovic and Sussmann [9].

THEOREM 3.1. Suppose there exists a $C^{1}$ function

$$
\xi=k(\zeta)
$$

with $k(0)=0$ such that the origin $\zeta=0$ of the system

$$
\dot{\zeta}=\phi(\zeta, k(\zeta))
$$

is globally asymptotically stable. Furthermore, let $V_{0}(\zeta)$ be a Lyapunov function for $(10)$, and define $\psi: \mathbb{R}^{n-1} \times \mathbb{R} \rightarrow \mathbb{R}^{n-1}$ by

$$
\psi(\zeta, \xi) \triangleq \frac{1}{\xi-k(\zeta)}[\phi(\zeta, \xi)-\phi(\zeta, k(\zeta))]
$$

Then the feedback control law

$$
v(\zeta, \xi)=k(\zeta)-\xi+\frac{\partial k(\zeta)}{\partial \zeta} \phi(\zeta, \xi)-\frac{\partial V_{0}(\zeta)}{\partial \zeta} \psi(\zeta, \xi)
$$

globally asymptotically stabilizes the origin $\zeta=0, \xi=0$ of (7), (8). Moreover, a Lyapunov function for the closed loop system is

$$
V(\zeta, \xi)=V_{0}(\zeta)+\frac{1}{2}[\xi-k(\zeta)]^{2}
$$

with time derivative

$$
\left.\dot{V}(\zeta, \xi)=\frac{\partial V_{0}(\zeta)}{\partial \zeta} \phi(\zeta, k(\zeta))-[\xi-k(\zeta))\right]^{2}
$$

Note that, according to l'Hôpital's rule, $\psi(\zeta, \xi)=\partial \phi(\zeta, k(\zeta)) / \partial \xi$, when $\xi=k(\zeta)$.

\section{Stabilizing Control Laws for the Oscillating Eccentric Rotor}

In this section, we synthesize control laws for the oscillating eccentric rotor that globally despin the rotor and bring the translational motion of the cart to rest in the case $F_{d}=0$. That is, we design control laws that globally asymptotically stabilize (6) to the equilibrium state $x=0$, where $f(x)$ and $g(x)$ are defined in Section 2. This is accomplished by first using partial feedback linearization to transform (6) into a system having cascade form. Then we iteratively apply Theorem 3.1 to synthesize control laws for the system in cascade form. Control laws for the original systems are then obtained from the reverse transformation.

Since the linearization of (6) about the origin is controllable, one can easily design linear control laws that locally asymptotically stabilize (6) to the origin. Linear control laws, however, are guaranteed to asymptotically stabilize (6) only for initial conditions sufficiently near the origin. Since we are interested in the despin problem for which none of the states are initially small, we seek nonlinear control laws that stabilize (6) for arbitrary initial conditions. 


\subsection{Partial FeEdBaCK Linearization}

To obtain globally asymptotically stabilizing control laws, we first check feedback linearizability of (6) [14]. It is straightforward to verify that $\left[g(x), \operatorname{ad}_{f}^{2} g(x)\right] \notin \Delta_{2}(x) \triangleq$ $\operatorname{span}\left\{g(x), \operatorname{ad}_{f} g(x), \operatorname{ad}_{f}^{2} g(x)\right\}$, where standard expressions for Lie brackets have been used $[5,14]$. Hence, $\Delta_{2}(x)$ is not involutive [5]. Consequently, (6) is not feedback linearizable [14].

Next we consider partial feedback linearization $[1,10]$. Since $\Delta_{1}(x)=\bar{\Delta}_{1}(x)$ and $\operatorname{dim} \Delta_{1}(x)=2$, where $\Delta_{1}(x) \triangleq \operatorname{span}\left\{g(x), \operatorname{ad}_{f} g(x)\right\}$, and $\bar{\Delta}_{1}(x)$ is the smallest involutive distribution which contains $\Delta_{1}(x)$ [1], it follows that the dimension of the largest linearizable subsystem is two [11]. To transform (6) into a cascade form with linear part having maximal dimension, we apply the following procedure. Since $\Delta_{1}(x)$ is involutive, it follows from the Frobenius theorem $[5,14]$ that solutions to the partial differential equations

$$
L_{g} z_{i}(x)=0, \quad L_{\mathrm{ad}_{f} g} z_{i}(0), \quad i=1,2,
$$

exist, where

$$
\operatorname{ad}_{f} g(x)=\frac{1}{1-\varepsilon^{2} \cos ^{2} x_{3}}\left[\begin{array}{c}
\varepsilon \cos x_{3} \\
-\varepsilon x_{4} \sin x_{3} \\
-1 \\
0
\end{array}\right]
$$

One set of solutions to these equations is

$$
\begin{aligned}
& z_{1}(x)=x_{1}+\varepsilon \sin x_{3}, \\
& z_{2}(x)=x_{2}+\varepsilon x_{4} \cos x_{3} .
\end{aligned}
$$

The new state variables in the linearized part of the transformed system can be obtained by letting $y_{1}(x)$ be such that

$$
L_{g} y_{1}(x)=0, \quad L_{\mathrm{ad}_{f} g} y_{1}(x) \neq 0,
$$

and such that the vectors

$$
\left(\frac{\partial y_{1}}{\partial x}\right)^{T}, \quad\left(\frac{\partial z_{1}}{\partial x}\right)^{T}, \quad\left(\frac{\partial z_{2}}{\partial x}\right)^{T},
$$

are linearly independent [11]. The simplest solution to these constraints is $y_{1}(z)=x_{3}$. Letting

$$
y_{2}(x)=L_{f} y_{1}(x)=x_{4},
$$

one can rewrite equation (6) in terms of the new variables $\left(z_{1}, z_{2}, y_{1}, y_{2}\right)$ as

$$
\begin{aligned}
& \dot{z}_{1}=z_{2}, \\
& \dot{z}_{2}=-z_{1}+\varepsilon \sin y_{1}, \\
& \dot{y}_{1}=y_{2}, \\
& \dot{y}_{2}=v,
\end{aligned}
$$


where

$$
v=\frac{1}{1-\varepsilon^{2} \cos ^{2} x_{3}}\left(\varepsilon x_{1} \cos x_{3}-\varepsilon^{2} x_{4}^{2} \cos x_{3} \sin x_{3}\right)+\frac{1}{1-\varepsilon^{2} \cos ^{2} x_{3}} u
$$

is the transformed control input. Note that (22), (23) are of the form (7) with $\xi=y_{1}$, $\zeta=z=\left(z_{1}, z_{2}\right)^{T}$ and

$$
\phi(\zeta, \xi)=\phi\left(z, y_{1}\right)=\left[\begin{array}{c}
z_{2} \\
-z_{1}+\varepsilon \sin y_{1}
\end{array}\right]
$$

Solving for $u$ in terms of $v$ yields

$$
u=\left(1-\varepsilon^{2} \cos ^{2} x_{3}\right) v-\varepsilon x_{1} \cos x_{3}+\varepsilon^{2} x_{4}^{2} \cos x_{3} \sin x_{3} .
$$

Having transformed (6) into the form (22)-(25), we now seek a control law $v$ that stabilizes (22)-(25) to the origin $y_{1}=y_{2}=z_{1}=z_{2}=0$, which corresponds to $x=0$ in (6).

\subsection{INTEGRATOR BACKSTEPPING}

In this subsection we synthesize a control law $v$ for (22)-(25) by iteratively applying Theorem 3.1. First, in Step 1, we view $y_{1}$ as the control input to (23) and seek a control law $y_{1}=k_{1}(z)$ that stabilizes (22) and (23). Then, in Step 2, we use Theorem 3.1 with $y_{2}$ as the control input for (24) to obtain a control law $y_{2}=k_{2}\left(z, y_{1}\right)$ that stabilizes (22)-(24). Finally, in Step 3, we use Theorem 3.1 again to derive a control law $v$ that stabilizes (22)-(25).

Step 1. Consider the problem of stabilizing (22), (23) with $y_{1}$ as the control input. To do this, let

$$
y_{1}=k_{1}(z) \triangleq-c_{0} \tan ^{-1} z_{2}
$$

where $0<c_{0}<2$, and consider the Lyapunov candidate

$$
V_{0}(z)=\frac{p_{0}}{2}\left(z_{1}^{2}+z_{2}^{2}\right)
$$

where $p_{0}>0$. Then it follows that

$$
\dot{V}_{0}(z)=-\varepsilon p_{0} z_{2} \sin \left(c_{0} \tan ^{-1} z_{2}\right)
$$

which is nonpositive. Next, consider the set $\mathcal{E}=\left\{z \in \mathbb{R}^{2}: \dot{V}_{0}(z)=0\right\}$. It is easy to see that $\mathcal{E}=\left\{z \in \mathbb{R}^{2}: z_{2}=0\right\}$. Consider $z_{2}(t)=0$ for $t \geq t_{1}$. From (23) and (29), we have $z_{1}(t)=\dot{z}_{2}(t)=0, t \geq t_{1}$. Hence, the largest invariant set in $\mathcal{E}$ is $\{0\}$. It thus follows from the LaSalle-Krasovskii theorem [14] that the states of the closed-loop system (22), (23) with $y_{1}$ defined in (29) approach the origin as $t \rightarrow \infty$. Finally, since $V_{0}(z) \rightarrow \infty$ as $\|z\| \rightarrow \infty$, it follows that the control law (29) globally asymptotically stabilizes (22), (23).

Step 2. We now use Theorem 3.1 to obtain a control law for (22)-(24) with $y_{2}$ as the control input. That is, we seek a feedback control law $y_{2}=k_{2}\left(z, y_{1}\right)$ such that (22)-(24) are globally asymptotically stable. From Step 1 and Theorem 3.1 we have $k(\zeta)=k_{1}(z)=-c_{0} \tan ^{-1} z_{2}$. A direct calculation shows that (10) is now

$$
\phi(\zeta, k(\zeta))=\phi\left(z, k_{1}(z)\right)=\left[\begin{array}{c}
z_{2} \\
-z_{1}-\varepsilon \sin \left(c_{0} \tan ^{-1} z_{2}\right)
\end{array}\right]
$$


while $\psi(\zeta, \xi)$ defined by $(11)$ is $\psi(\zeta, \xi)=\psi\left(z, y_{1}\right)$ and

$$
\psi\left(z, y_{1}\right)=\left[\begin{array}{c}
0 \\
\varepsilon\left[\sin y_{1}+\sin \left(c_{0} \tan ^{-1} z_{2}\right)\right] /\left(y_{1}+c_{0} \tan ^{-1} z_{2}\right)
\end{array}\right] .
$$

With the Lyapunov candidate

$$
V_{1}\left(z, y_{1}\right)=\frac{p_{0}}{2}\left(z_{1}^{2}+z_{2}^{2}\right)+\frac{p_{1}}{2}\left(y_{1}+c_{0} \tan ^{-1} z_{2}\right)^{2},
$$

where $p_{1}>0$, Theorem 3.1 yields the control law $y_{2}=k_{2}\left(z, y_{1}\right)$, where

$$
\begin{aligned}
k_{2}\left(z, y_{1}\right) \triangleq & -c_{1}\left(y_{1}+c_{0} \tan ^{-1} z_{2}\right)+c_{0} \frac{z_{1}-\varepsilon \sin y_{1}}{1+z_{2}^{2}} \\
& -\frac{\varepsilon p_{0} z_{2}}{p_{1}\left(y_{1}+c_{0} \tan ^{-1} z_{2}\right)}\left[\sin y_{1}+\sin \left(c_{0} \tan ^{-1} z_{2}\right)\right],
\end{aligned}
$$

and $c_{1}>0$. The time derivative of $V_{1}\left(z, y_{1}\right)$ along the closed-loop trajectory (22)-(24) with the control law $y_{2}$ defined by (35) is

$$
\dot{V}_{1}\left(z, y_{1}\right)=-\varepsilon p_{0} z_{2} \sin \left(c_{0} \tan ^{-1} z_{2}\right)-p_{1} c_{1}\left(y_{1}+c_{0} \tan ^{-1} z_{2}\right)^{2} .
$$

It is straightforward to check that the largest invariant set in $\left\{\left(z, y_{1}\right): \dot{V}_{1}\left(z, y_{1}\right)=0\right\}$ is $\left\{z=0, y_{1}=0\right\}$. Hence, the control $y_{2}$ in (35) globally asymptotically stabilizes (22)-(24) to $z=0, y_{1}=0$.

Step 3. Now we apply Theorem 3.1 to obtain a stabilizing control law for the entire system (22)-(25). Note that (22)-(24) and (25) are again in the cascade form of (7) and (8) with $\xi=y_{2}, \zeta=\left(z_{1}, z_{2}, y_{1}\right)^{T}$ and

$$
\phi(\zeta, \xi)=\phi\left(\zeta, y_{2}\right)=\left[\begin{array}{c}
z_{2} \\
-z_{1}+\varepsilon \sin y_{1} \\
y_{2}
\end{array}\right] \text {. }
$$

Furthermore, in Step 2 we have already established a $C^{1}$ function $y_{2}=k_{2}\left(z, y_{1}\right)$ with $k_{2}(0,0)=0$ that globally asymptotically stabilizes (22)-(24) and a Lyapunov function $V_{1}\left(z, y_{1}\right)$ defined in (34). Hence we apply Theorem 3.1 again to obtain the stabilizing control law

$$
\begin{aligned}
v(z, y)= & -c_{2}\left(y_{\dot{2}}-k_{2}\left(z, y_{1}\right)\right)+K_{1}\left(z_{2}\right) z_{2}+K_{2}\left(z, y_{1}\right)\left(-z_{1}+\varepsilon \sin y_{1}\right) \\
& +K_{3}\left(z, y_{1}\right) y_{2}-\frac{p_{1}}{p_{2}}\left(y_{1}+c_{0} \tan ^{-1} z_{2}\right),
\end{aligned}
$$

for (22)-(25), where $y=\left(y_{1}, y_{2}\right)^{T}, c_{2}>0, k_{2}\left(z, y_{1}\right)$ is defined in (35), and $K_{1}\left(z_{2}\right), K_{2}\left(z, y_{1}\right)$, $K_{3}\left(z, y_{1}\right)$ are defined by

$$
\begin{aligned}
K_{1}\left(z_{2}\right) \triangleq & \frac{c_{0}}{1+z_{2}^{2}}, \\
K_{2}\left(z, y_{1}\right) \triangleq & -\frac{\varepsilon p_{0}}{p_{1}\left(y_{1}+c_{0} \tan ^{-1} z_{2}\right)}\left[\sin y_{1}+\sin \left(c_{0} \tan ^{-1} z_{2}\right)+c_{0} z_{2} \frac{\cos \left(c_{0} \tan ^{-1} z_{2}\right)}{1+z_{2}^{2}}\right] \\
& -\frac{c_{0} c_{1}}{1+z_{2}^{2}}-2 c_{0} z_{2} \frac{z_{1}-\varepsilon \sin y_{1}}{\left(1+z_{2}^{2}\right)^{2}}+\frac{\varepsilon p_{0} c_{0}}{p_{1}} \frac{z_{2}\left[\sin y_{1}+\sin \left(c_{0} \tan ^{-1} z_{2}\right)\right]}{\left(1+z_{2}^{2}\right)\left(y_{1}+c_{0} \tan ^{-1} z_{2}\right)^{2}}
\end{aligned}
$$


and

$$
\begin{aligned}
K_{3}\left(z, y_{1}\right) \triangleq & -c_{1}-\frac{\varepsilon c_{0} \cos y_{1}}{1+z_{2}^{2}}-\frac{\varepsilon p_{0}}{p_{1}} \frac{z_{2} \cos y_{1}}{y_{1}+c_{0} \tan ^{-1} z_{2}} \\
& +\frac{\varepsilon p_{0}}{p_{1}} \frac{z_{2}\left[\sin y_{1}+\sin \left(c_{0} \tan ^{-1} z_{2}\right)\right]}{\left(y_{1}+c_{0} \tan ^{-1} z_{2}\right)^{2}}
\end{aligned}
$$

The Lyapunov function that guarantees global asymptotic stability of the closed-loop system (22)-(25) with $v$ defined in (37) is

$$
V_{2}(z, y)=\frac{p_{0}}{2}\left(z_{1}^{2}+z_{2}^{2}\right)+\frac{p_{1}}{2}\left(y_{1}+c_{0} \tan ^{-1} z_{2}\right)^{2}+\frac{p_{2}}{2}\left[y_{2}-k_{2}\left(z, y_{1}\right)\right]^{2},
$$

where $p_{2}>0$. Note that $V_{2}(z, y)$ is proper and positive definite. The time derivative of $V_{2}(z, y)$ is

$$
\begin{aligned}
\dot{V}_{2}(z, y)= & -\varepsilon p_{0} z_{2} \sin \left(c_{0} \tan ^{-1} z_{2}\right) \\
& -p_{1} c_{1}\left(y_{1}+c_{0} \tan ^{-1} z_{2}\right)^{2}-p_{2} c_{2}\left[y_{2}-k_{2}\left(z, y_{1}\right)\right]^{2},
\end{aligned}
$$

which is negative semi-definite. It can be shown that along the closed-loop trajectories the largest invariant set in $\left\{(z, y): \dot{V}_{2}(z, y)=0\right\}$ is $\{z=y=0\}$. Since $V_{2}(z, y)$ is proper, it follows that $v$ given by (37) globally asymptotically stabilizes the system (22)-(25). Note that $k_{2}\left(z, y_{1}\right), K_{2}\left(z, y_{1}\right)$ and $K_{3}\left(z, y_{1}\right)$ are continuous for all their arguments.

Finally, the feedback control law $u$ that globally asymptotically stabilizes the original system (6) to the origin is obtained by substituting (37) into (28).

REMARK 1. In Step 1, we chose $k_{1}(z)$ in (29) in terms of the inverse tangent function. Other choices of $k_{1}(z)$ may be made in accordance with Theorem 3.1. It can be shown that if $k_{1}(z)$ is a $C^{1}$ bounded function with $k_{1}(0)=0$ and $z_{2} k_{1}(z)<0, z \in \mathbb{R}^{2}$, then $y_{1}=k_{1}(z)$ globally asymptotically stabilizes (22), (23).

REMARK 2. The six parameters $p_{0}, p_{1}, p_{2}, c_{0}, c_{1}$ and $c_{2}$ which appear in the control law are allowed to vary over specified ranges $p_{0}>0, p_{1}>0, p_{2}>0,0<c_{0}<2, c_{1}>0$ and $c_{2}>0$. All six parameters appear explicitly in the control law and time derivative of the Lyapunov function and thus affect the convergence rate, the transient response and the required control effort.

In the next section we choose values for these parameters and perform numerical simulations.

\section{Simulation Results}

For simulation we consider the dimensionless equations (5) and assume $\varepsilon=0.1$. We first consider the stabilization problem in which there is no external force applied to the cart, that is, $F_{d}=0$. In particular, we numerically show that the feedback control law $u$ in (28) with $v$ defined in (37) stabilizes (6) to the origin. Two sets of control parameters are chosen for the control law, specifically,

Controller 1: $p_{0}=p_{1}=p_{2}=c_{0}=c_{1}=c_{2}=1$;

Controller 2: $p_{0}=10, p_{1}=0.1, c_{0}=1.9, p_{2}=c_{1}=c_{2}=1$. 


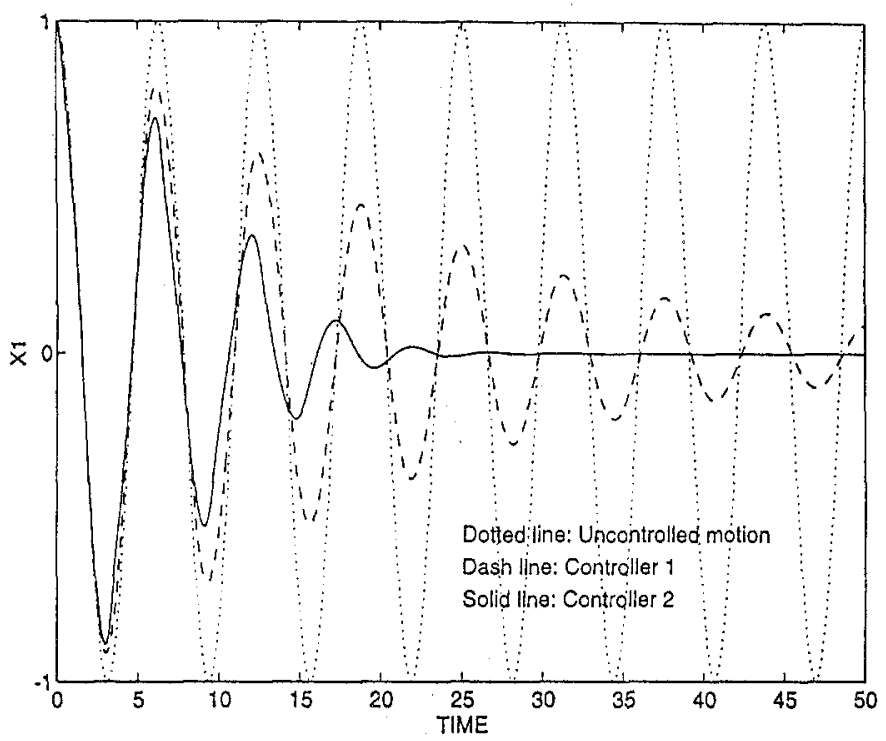

Fig. 2. Time history of cart translation $x_{1}$ of the uncontrolled and controlled motions with $x(0)=(1,0,0,0)^{T}$.

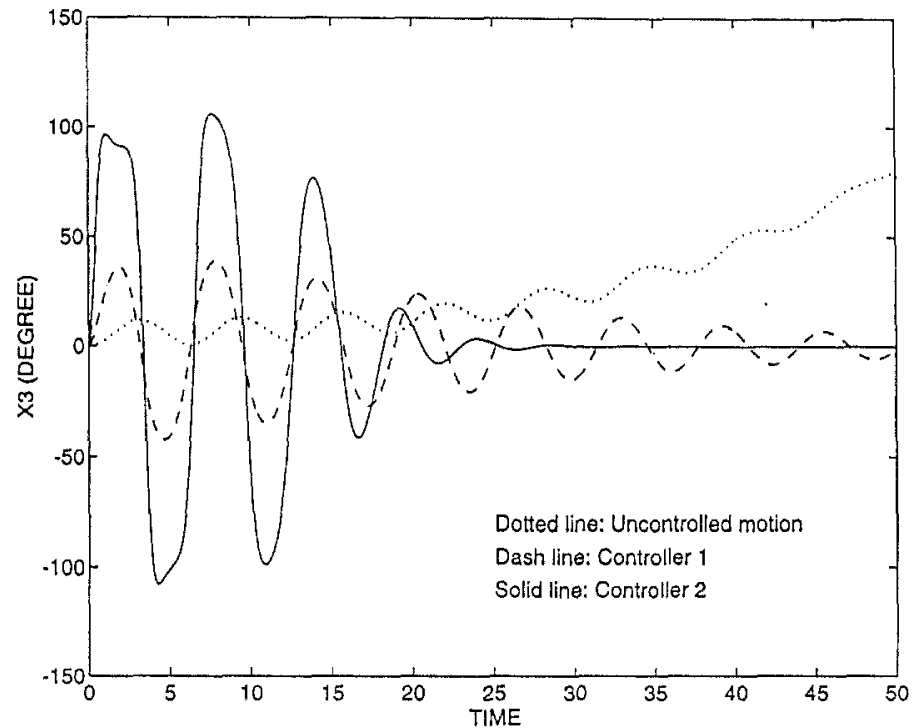

Fig. 3. Time history of eccentric mass rotation $x_{3}$ of the uncontrolled and controlled motions with $x(0)=(1,0,0,0)^{T}$.

In the first example, we consider the case in which the cart has an initial displacement while the disk has zero initial angular velocity, that is, $x(0)=(1,0,0,0)^{T}$. Figures 2 and 3 illustrate the cart translation $x_{1}(\tau)$ and eccentric mass rotation $x_{3}(\tau)$ of the uncontrolled and controlled motions. Note that the rotor is free to spin for the uncontrolled system. Figure 4 shows the control torque $u$ for Controllers 1 and 2 with respect to time. From Figures 2 and 3 it can be seen that the translational motion of the uncontrolled system is oscillatory with fixed amplitude and frequency while the rotor angle is increasing. For the controlled system, both Controllers 1 and 2 drive the translational and rotational oscillations to rest. Note that 


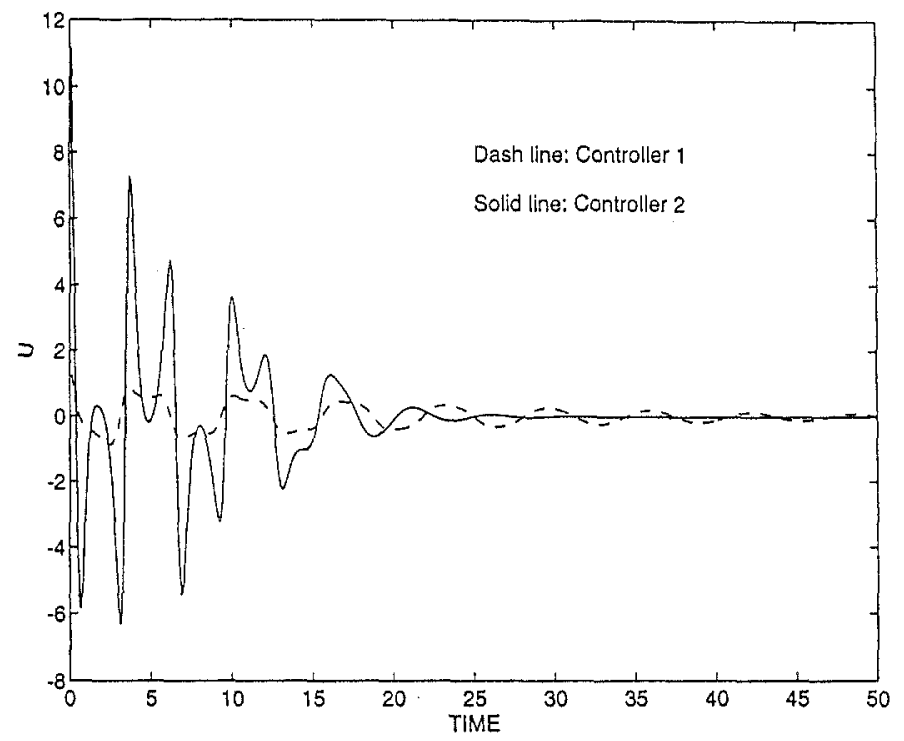

Fig. 4. Control torque $u$ for Controller 1 and 2 with $x(0)=(1,0,0,0)^{T}$.

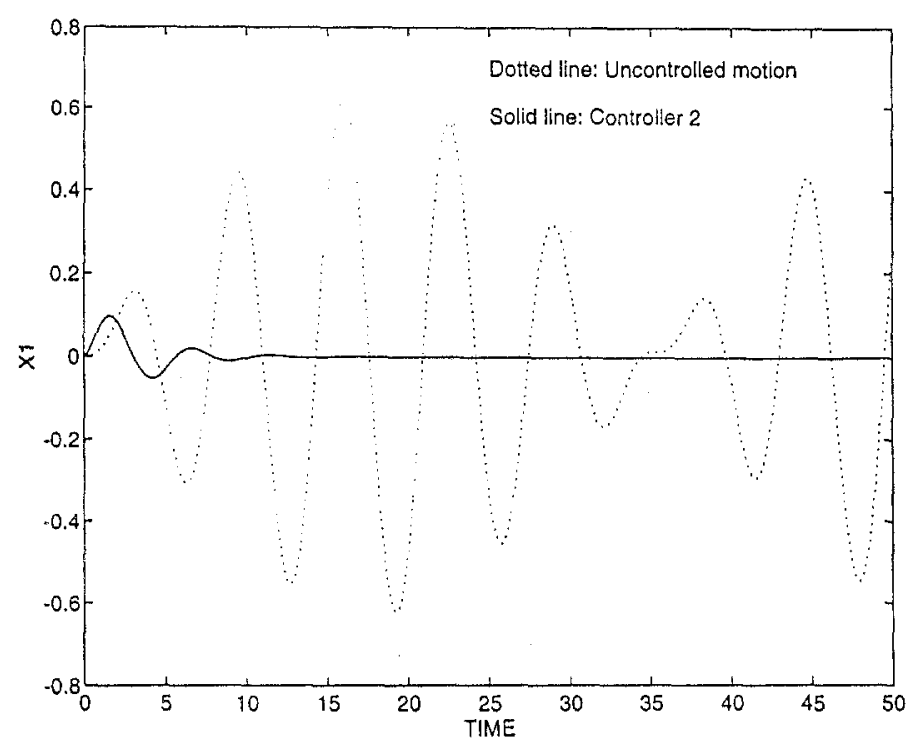

Fig. 5. Time history of cart translation $x_{1}$ of the uncontrolled and controlled motions with $x(0)=(0,0,0,1)^{T}$.

Controller 2 drives the motion to rest faster than Controller 1 . Since this was found to be the case for all initial conditions tested, Controller 1 will not be considered further.

Next we consider the case in which the cart is at rest while the desk has angular velocity equal to the resonance frequency of the translational motion, that is, $x(0)=(0,0,0,1)^{T}$. Figures 5,6 and 7 show the cart translation $x_{1}(\tau)$, eccentric mass rotation $x_{3}(\tau)$, and angular velocity $x_{4}(\tau)$ of the uncontrolled and controlled motions. It can be seen in Figure 7 that the angular velocity $x_{4}(\tau)$ remains close to 1 for the uncontrolled motion, which shows that the eccentric mass lies within the resonance capture zone. However, as shown in Figures 5,6 and 


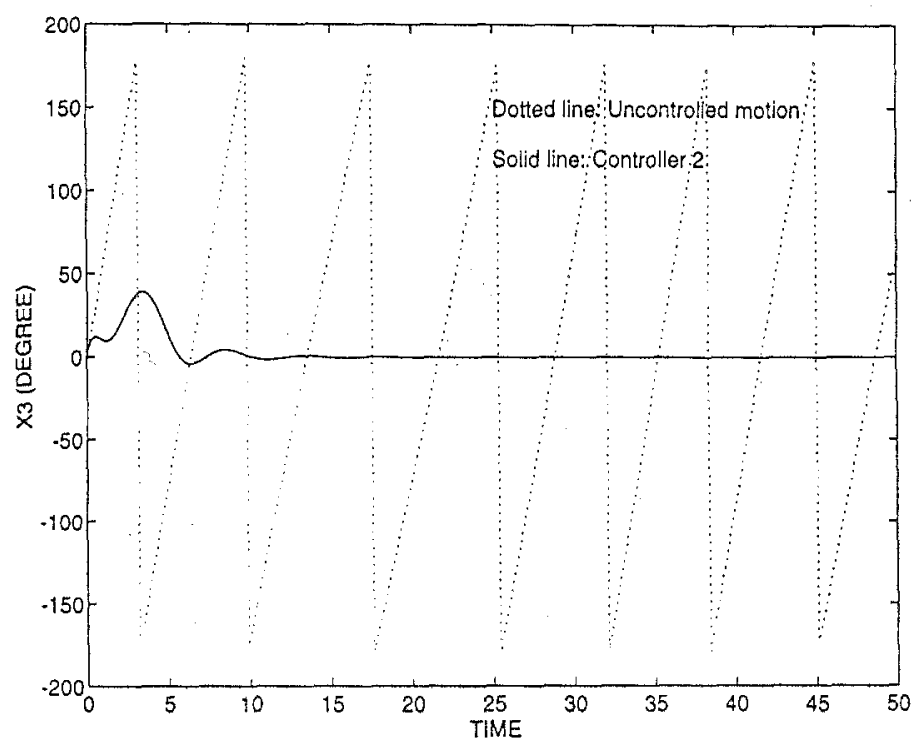

Fig. 6. Time history of eccentric mass rotation $x_{3}$ of the uncontrolled and controlled motions with $x(0)=(0,0,0,1)^{T}$.

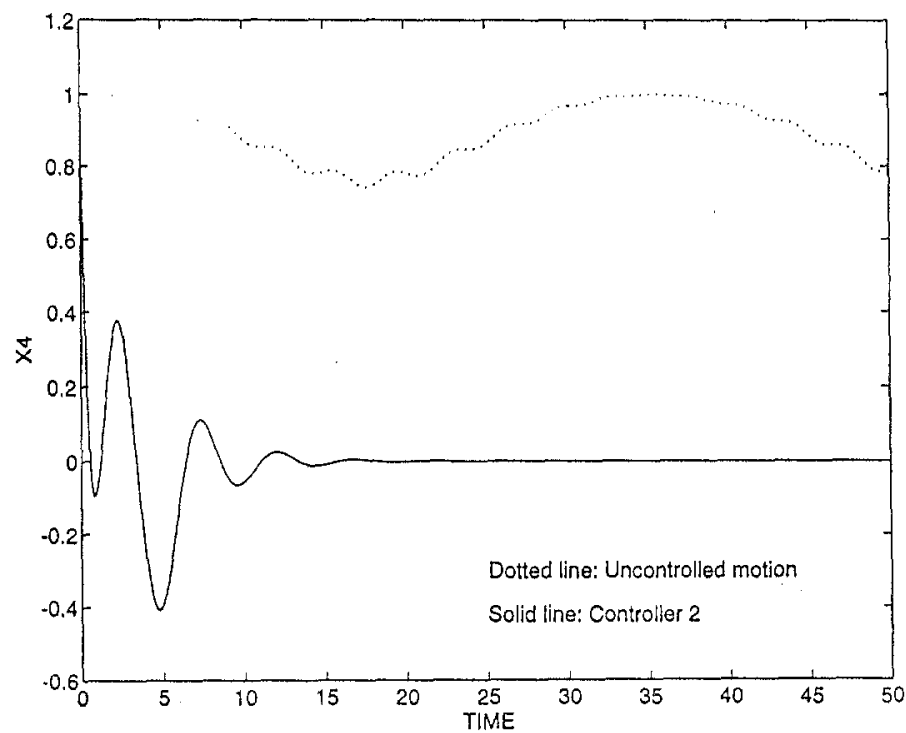

Fig. 7. Time history of eccentric mass angular velocity $x_{4}$ of the uncontrolled and controlled motions with $x(0)=(0,0,0,1)^{T}$.

7 , the states of the controlled motion approach the origin very rapidly, while the control torque $u$ (not shown) decrease rapidly with the maximum torque $|u|=3.2$ needed at $\tau=0$.

Next, we consider the case in which there are external disturbances applied to the cart and show that the control law, although designed without regard to disturbances, attenuates the forced translational oscillation. We thus consider (5) with sinusoidal forcing function

$$
F_{d}=A_{F} \sin \omega_{F} \tau
$$




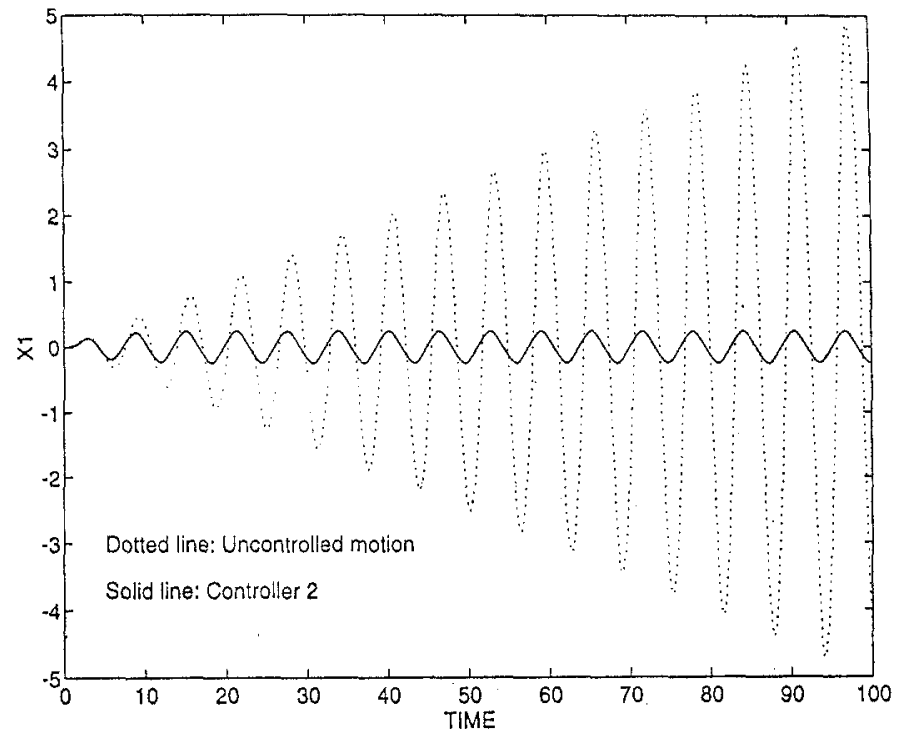

Fig. 8. Time history of cart translation $x_{1}$ of the uncontrolled and controlled motions for resonant forcing $\omega_{F}=1$ at amplitude $A_{F}=0.1$ with $x(0)=(0,0,-\pi / 2,0)^{T}$.

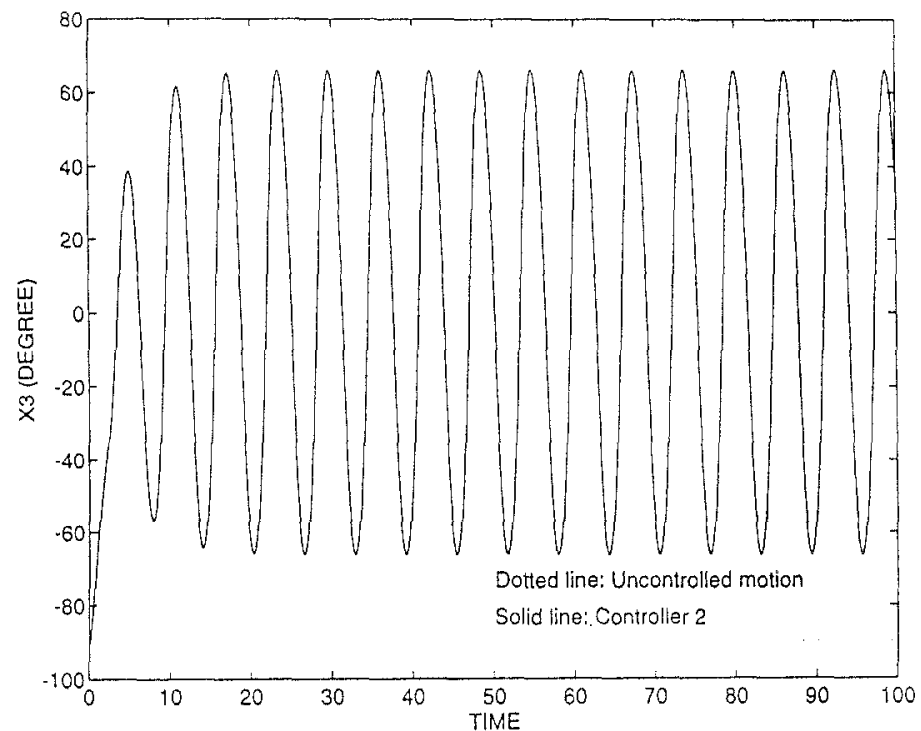

Fig. 9. Time history of eccentric mass rotation $x_{3}$ of the uncontrolled and controlled motions for resonant forcing $\omega_{F}=1$ at amplitude $A_{F}=0.1$ with $x(0)=(0,0,-\pi / 2,0)^{T}$.

where $A_{F}, \omega_{F}$ are the amplitude and frequency of the external forcing. We also assume that the unbalanced mass is initially aligned with the spring and that both the cart and the disk are at rest, that is, $x(0)=(0,0-\pi / 2,0)^{T}$. Figures 8 and 9 show the translation $x_{1}(\tau)$ and rotations $x_{3}(\tau)$ of the uncontrolled and controlled motions for resonant forcing $\omega_{F}=1$ at amplitude $A_{F}=0.1$. It can be seen from Figures 8 and 9 that the controller attenuates the amplitude of the translational oscillation by preventing the linear amplitude growth of the uncontrolled motion. Figure 10 shows the time history of the control torque. 


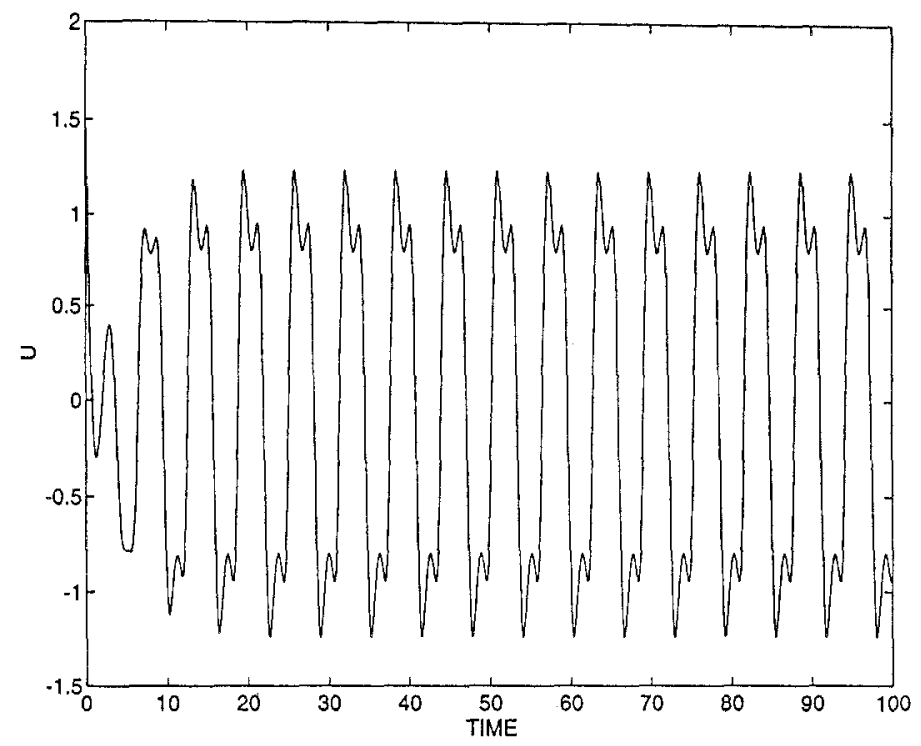

Fig. 10. Control torque $u$ for resonant forcing $\omega_{F}=1$ at amplitude $A_{F}=0.1$ with $x(0)=(0,0,-\pi / 2,0)^{T}$.

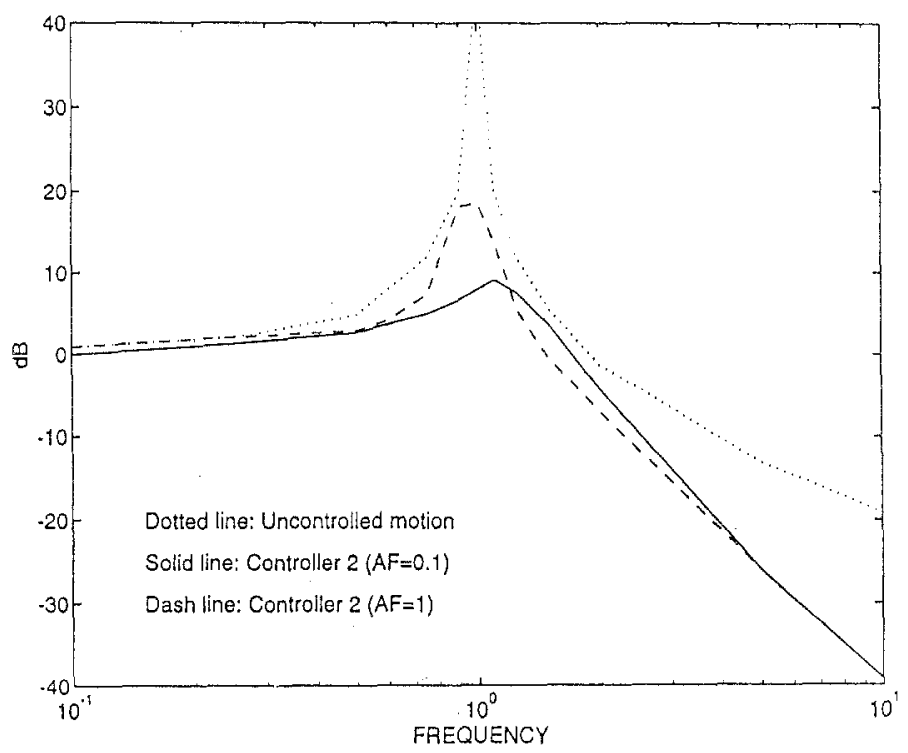

Fig. 11. Amplitude-dependen: frequency response of the translational oscillation of the uncontrolled and controlled motions under sinusoidal forcing with amplitudes $A_{F}=0.1$ and $A_{F}=1$.

Finally, Figure 11 shows the amplitude-dependent frequency response of the translational oscillation of the uncontrolled and controlled motions under sinusoidal forcing with amplitudes $A_{F}=0.1$ and $A_{F}=1$. Controller 2 is used for the controlled motion. The vertical axis of Figure 11 is the ratio of the steady-state amplitude of the translational oscillation to the forcing amplitude. The forcing frequency ranges from 0.1 to $10 \mathrm{rad} / \mathrm{sec}$. It is observed that the controller is most effective near and above resonance and is more effective for smaller disturbance amplitude except for frequencies near twice the resonance. 


\section{Conclusions}

We have derived a globally asymptotically stabilizing control law for the oscillating eccentric rotor that uses torque control to simultaneously bring both translational and rotational motions to rest. Six independent parameters in the control law allow adjustment of the closed-loop response. In addition, numerical simulations show that the control law, although designed without regard to disturbances, can attenuate sinusoidal disturbances over a broad frequency range.

In a recent paper [2], we explored an application of this work, a rotational actuator for suppressing translational motion. Two control laws were compared: (i) the control based on integrator backstepping given herein and (ii) an active implementation of a passive control law. The passive control law emulates a vibration absorber that damps out both rotational and translational energy. Note that a simple rotational rate damper cannot globally asymptotically stabilize the translational motion. The two approaches have different advantages and disadvantages that are currently being investigated; interested readers should consult [2].

\section{References}

1. Bacciotti, A., Local Stabilizability of Nonlinear Control Systems, Series on Advances in Mathematics for Applied Sciences, Vol. 8, World Scientific, Singapore, 1992.

2. Bupp, R., Wan, C.-J., Coppola, V. T., and Bernstein, D. S., 'Design of a rotational actuator for global stabilization of translational motion', in Active Control of Vibration and Noise, ASME Winter Meeting, DE-Vol. 75, 1994, pp. 449-456.

3. Ewan-Iwanowski, R. M., Resonance Oscillations in Mechanical Systems, Elsevier, Amsterdam, 1976.

4. Hall, C. D. and Rand, R. H., 'Spinup dynamics of axial dual-spin spacecraft', Journal of Guidance, Control and Dynamics 17(1), 1994, 30-37.

5. Isidori, A., Nonlinear Control Systems, Second Edition, Springer-Verlag, New York, 1989.

6. Kanellakopoulos, I., Kokotovic, P. V., and Morse, A. S., 'A toolkit for nonlinear feedback design', Systems and Control Letters 18, 1992, 83-92.

7. Kinsey, R. J., Mingori, D. L., and Rand, R. H., 'Nonlinear controller to reduce resonance effects during despin of a dual-spin spacecraft through precession phase lock', in Proceedings of the Conference on Decision and Control, Tucson, AZ, December, 1992, 3025-3030.

8. Kokotovic, P. V., 'The joy of feedback: nonlinear and adaptive', IEEE Control Systems Magazine, June, 1992, 7-17.

9. Kokotovic, P. V. and Sussmann, H. J., 'A positive real condition for global stabilization of nonlinear systems', Systems and Control Letters 13, 1989, 125-133.

10. Marino, R., 'On the largest feedback linearizable subsystem', Systems and Control Letters 6, 1984, 345-352.

11. Marino, R., 'High gain stabilization and partial feedback linearization', in Proceedings of the Conference on Decision and Control, Athens, Greece, December, 1986, 209-213.

12. Rand, R. H., Kinsey, R. J., and Mingori, D. L., 'Dynamics of spinup through resonance', International Journal of Non-Linear Mechanics 27, 1992, 489-502.

13. Sontag, E. D. and Sussmann, H. J., 'Further comments on the stabilizability of the angular velocity of a rigid body', Systems and Control Letters 12, 1989, 213-217.

14. Vidyasagar, M., Nonlinear Systems Analysis, Second Edition, Prentice Hall, Englewood Cliffs, NJ, 1993.

15. Yee, R. K., 'Spinup dynamics of a rotating system with limiting torque', Master's Thesis, UCLA, 1981. 